\section{Der überforderte Stakeholder}

\author{
Externe Anspruchsgruppen brauchen Informationen, um die Unternehmenspoli- \\ tik beeinflussen zu können. Sozial-ökologische Unternehmensbewertung kann \\ diese zur Verfügung stellen. Dies ist jedoch alles andere als einfach. Die hier- \\ für nötigen Informationen durchlaufen selbst vielfache Filterprozesse, die die \\ Gültigkeit von Bewertungen einschränken. Erst aus einer Analyse dieser Gren- \\ zen lassen sich Anforderungen an einen wirksamen sozial-ökologischen Bewer- \\ tungsprozess ableiten.
}

$\mathrm{W}$ Von Jürgen Freimann Unternehmen „über das Geld hinaus“ zu lenken? Einige Sozial- und Wirtschaftswissenschaftler schlagen folgende Vermittlung vor: Soziale und ökologische Belange seien schon immer im notwendigen Interessenausgleich zwischen den involvierten Akteuren enthalten, und daher im wohlverstandenen Eigeninteresse beachtenswert. Ihnen gilt nicht von ungefähr das Anspruchsgruppen- oder Stakeholder-Modell als liebstes Grundmodell der Unternehmenspolitik. Denn es modelliert zwangsläufige Vielfalt von Einflüssen und Orientierungen auf Grundlage einer entsprechenden Vielfalt von externen und internen Einflussnehmern, wo die zynische Vernunft des neoklassischen Mainstreams auch in seinen modernen Varianten über die monetäre Einfalt nicht hinauskommt.

Dem wirkt allerdings der Zug zur Globalisierung der Wirtschaft entgegen, der seit dem Bankrott des Realsozialismus als Universalisierung neoliberaler Orientierungen daherkommt. Er scheint den traditionellen Orientierungen - nun in Gestalt des shareholder value - als einzig relevantem Bewertungskriterium für den Unternehmenserfolg zu einer umfassenden Renaissance zu verhelfen.

Bei näherem Hinsehen entpuppt sich die moderne Orientierung an dieser Maßgröße jedoch als weit mehr sozial aufgeladen als die tradierte Gewinnmaximierung. Denn an Einflussfaktoren für die tatsächliche Höhe des shareholder value - misst man ihn einmal vereinfachend am Kurswert der Kapitalanteile - wird eine Vielzahl höchst differenter Variablen wirksam: Währungs- und Konjunktureinflüsse ebenso wie das allgemeine politische Klima einer Wirtschaftsregion, Stimmungen und Erwartungen institutio- neller und privater Anleger, das Image und die Kommunikationserfolge des Unternehmens sind nur einige davon. In der Börsenperformance eines Unternehmens kann sich sogar explizit dessen soziales und ökologisches Engagement niederschlagen, wenn dies über ein entsprechendes Rating den Anlegern nahegebracht wird und bei diesen auf Akzeptanz stößt.

Im Gegentrend zur so beschriebenen Renaissance der ,reinen“ Ökonomie im Verhältnis zwischen Unternehmen auf der einen, dem Staat, Verbänden und anderen Repräsentanten der Öffentlichkeit auf der anderen Seite lassen sich neuerdings häufiger Formen des diskursiven Konfliktausgleichs beobachten. Diese Erscheinungen deuten darauf hin, dass offensichtlich das Stakeholder-Modell doch nicht nur auf dem Wunschdenken sozial-ökologisch infizierter wissenschaftlicher Traumtänzer aufgebaut ist.

Nun sagt die Existenz von runden Tischen mit Unternehmensbeteiligung allein noch nichts über die faktische Durchsetzungsmacht der daran Beteiligten aus. Im Gegenteil darf vermutet werden, dass die Position zumindest der multinationalen Unternehmen angesichts glaubhafter Abwanderungsoptionen eher stärker geworden ist, die runden Tische also vielleicht sogar als Zeichen der gewachsenen Ohnmacht externer politischer Einflussnehmer auf Unternehmen gelten müssen.

Dennoch können und wollen die Unternehmen sich offensichtlich nicht völlig aus ihrer sozialen Verankerung lösen. Unternehmen bleiben soziale Organisationen, und auch an den Märkten, an denen in immer größerem Maße nahezu identische Produkte angeboten werden, spielen Imagefaktoren und damit soziale und ökologische Wertungen eine wachsende Rolle.
Angesichts der beschriebenen Entwicklungen stellt sich die Frage nach den Informationsgrundlagen für die verschiedenen Stakeholder (einschließlich der Marktpartner). Wie valide und brauchbar sind diese für die Wahrung von Einfluss- und Durchsetzungschancen in derart gewandelten Prozessen der corporate governance? Nach den Chancen und Grenzen der systematischen sozial-ökologischen Information von unternehmensexternen Stakeholdern zu fragen ist also sinnvoll und notwendig.

\section{Information und Interesse}

Unternehmensinformationen stammen überwiegend von den Unternehmen selbst. Sie werden in der Regel von PR-Profis - oder zumindest nicht ohne deren Mitwirkung - aus freien Stücken oder auf Anfrage namens des Unternehmens publiziert. Sie richten sich an die Öffentlichkeit allgemein oder an bestimmte Zielgruppen, erreichen jedoch diese zumeist nicht direkt, sondern werden von verschiedenen Informationsvermittlern bearbeitet und transportiert. Schließlich bedürfen sie, um anzukommen, der Rezeption durch die Adressaten.

Auf allen Stufen dieses Informations-Erstellungs-, Bearbeitungs- sowie Rezeptionsprozesses sind spezifische Interessen der jeweiligen Akteure im Spiel. Der Informationsgenerierer Unternehmen will zumeist vor allem ein positives Bild von sich zeichnen, er tendiert dazu, potenziell negative Nachrichten eher zu verschweigen. Die Vermittler selektieren und bearbeiten die empfangenen Informationen zur Weitergabe nach Maßgabe ihrer spezifischen Interessen. Beispielsweise möchten sie Aufmerksamkeit erregen und Leser oder Seher binden. Die Empfänger suchen, selektieren und bewerten Informationen nach ihrer aktuellen Interessenlage, beispielsweise der Bedeutung und der Seriosität, die sie den Informationen beimessen.

Auf Grund der Vielfalt dieser interessenbedingten Einflüsse muss der Anspruch an die Objektivität und Glaubwürdigkeit von Unternehmensinformationen grundsätzlich relativiert werden: Von Interesseneinflüssen freie und in diesem Sinne objektive Information kann es nicht geben.

\section{Informationsflut}

und Informationsasymmetrie

Vor nicht allzu langer Zeit gab es noch zwei Fernseh- und wenige Hörfunkprogramme sowie eine überschaubare Zahl von Printmedien und Unternehmenspublikationen, und darin eine 
noch viel überschaubarere Menge von unternehmenspolitisch relevanten Informationen. Seither ist deren Menge immens gewachsen, was zugleich die Wahrnehmungsfähigkeit der Menschen reduziert hat. Verstärkt wird diese Entwicklung noch dadurch, dass bestimmte - und zwar vor allem die (unternehmens-)politisch irrelevanten - Informationen in ihrem Reiz- und Aufmerksamkeitscharakter immer schriller dargeboten werden. Sie legen den Menschen zu ihrem eigenen Schutz Verhaltensweisen zur Informationsabwehr nahe. Diesen fallen dann jedoch die weniger aufmerksamkeitsheischenden Informationen eher zum Opfer als andere. Daraus folgt im hier behandelten Kontext vor allem, dass der Grad der Selektivität, mit dem wir Informationen außerhalb unseres engeren Arbeits- und Lebenszusammenhangs aufnehmen, zugenommen hat. Nur die eng entscheidungsrelevanten Informationen (oder was wir dafür halten) nehmen wir in der Regel noch wahr und suchen vielleicht sogar danach.

Diese Tatsache führt dazu, dass immer mehr Informationsverdichtung vorgenommen wird. Hochaggregierte Kennzahlen oder Label treten an die Stelle differenzierter Informationen, die kaum jemand mehr aufzunehmen in der Lage ist. Vertrauen in die Validität der Informationsaggregate muss das mangelnde Wissen um Möglichkeiten und Grenzen ihrer Aussagekraft ersetzen. Nur noch wenige Experten - in der Regel diejenigen, die die Aggregate erstellen verfügen über Detailwissen über deren Inhalte und Konstruktionsmerkmale. Den Adressaten, die die Aggregate zur Grundlage für ihre wirtschaftlichen oder politischen Entscheidungen machen (müssen), bleibt vielfach kaum mehr übrig als zu glauben, dass sie auf einer solchen Entscheidungsgrundlage ,schon irgendwie das Richtige tun".

\section{Information und Systemcodes}

In diesem Zusammenhang scheint es geboten, an die Thesen der Systemtheorie zu erinnern. Danach sind Menschen ebenso wie soziale Institutionen geschlossene selbstreferenzielle Systeme, die geleitet durch ihre systeminternen Muster und Merkmale Realität konstruieren. Realität wird so beobachterabhängig.

Soziale Systeme verfügen nun aber über unterschiedliche Codes, das System Wirtschaft über andere als die Systeme Recht und Politik. Zwischen den Systemen sind Informationsaustausch und Verständigung zumindest eingeschränkt.
Soziale und ökologische Folgen wirtschaftlicher Verhaltensweisen sind im System Wirtschaft nur über den Preismechanismus als dem wirtschaftlichen Systemcode wahrnehm- und handhabbar. Schlagen sie sich darin nicht nieder, sind sie für das System irrelevant.

Insbesondere für solche Informationen, die verschiedene soziale Teilsysteme durchlaufen müssen, um wirtschaftliche oder politische Handlungsrelevanz zu bekommen, folgt daraus fast schon die Notwendigkeit der Verstümmelung. Dies gilt gerade für soziale und ökologische Informationen, die zumeist im System Wissenschaft erzeugt und von dessen Codes geprägt werden.

Auch wenn in der realen Welt soziale Subjekte durch ihre regelmäßig vorhandene Einbindung in verschiedene soziale Teilsysteme durchaus „mehrsprachig“ kommunizieren können, setzt die skizzierte Informationsverstümmelung der Möglichkeit valider Informationsgrundlagen für die unternehmensexternen Stakeholder enge Grenzen.

\section{Halbvoll oder halbleer?}

Es gibt mithin keine objektiven, von verzerrenden Einflüssen unberührten Informationen und schon gar keine über Unternehmen. Darüber hinaus sind zumeist immer noch unterschiedliche Interpretationen ein und derselben Information möglich. Es ist immer auch noch eine subjektive Komponente im Spiel, deren Wirkung von den Kontextbedingungen bestimmt wird und die zudem im Zeitablauf Wandlungen unterliegt. Vielen Unternehmen, die sich einem Öko-Audit unterzogen haben, galt nachher die Einhaltung der einschlägigen Rechtsvorschriften des Umweltschutzes als beachtliche Leistung. Vorher kannten sie vielfach noch nicht einmal alle für sie zutreffenden Gesetze und wähnten sich daher vielleicht - wie die meisten Verkehrsteilnehmer - selbstverständlich im Einklang mit allen Vorschriften. Während jedoch beim halbvollen beziehungsweise halbleeren Glas immerhin die Silbe „halb“ durch die Größe des Glases hinreichend genau bestimmt ist, fehlt im Bereich der sozial-ökologischen Bewertung selbst hierfür vielfach die Bezugsgrundlage. Zweifellos ist die Halbierung des Abwasseraufkommens prima facie ein Erfolg des betrieblichen Umweltschutzes. Die Aussagekraft dieses Indikators basiert jedoch entscheidend auf weiteren Kontextinformationen: Von welcher Ausgangsbasis wurde ausgegangen; was sind die Ursachen für den beobachteten Effekt, wie hoch ist der Verschmutzungsgrad vorher und nachher, welche Entwicklung ist in den anderen Umweltmedien zu beobachten, wo liegen vergleichbare Wettbewerber und dergleichen? Dieses Interpretationsproblem besteht insbesondere bei Informationen über Art, Umfang und Wirksamkeit von Umweltmanagementaktivitäten.

Nicht zuletzt schaffen erst die Existenz und die Kenntnis von betrieblichen und gesamtwirtschaftlichen Zielsetzungen eine brauchbare Urteilsgrundlage. Gibt es eine konkrete politische Zielstellung zum Beispiel für das Ausmaß von Wasserverbrauch und umweltverträglichen Abwassereinträgen in einer Region, dann ist es sehr viel leichter, den notwendigen Beitrag verschiedener wirtschaftlicher Akteure zur Zielerreichung zu beziffern und ihren tatsächlichen Beitrag daran $\mathrm{zu}$ messen, als ohne derartige Ziele. Zumeist allerdings fehlen sie in der Praxis oder sind jedenfalls nicht konsensfähig.

\section{- Monetäre Bewertung oder nicht?}

Die wissenschaftliche Debatte um die Möglichkeiten und Grenzen der sozial-ökologischen Unternehmensbewertung wird seit Anbeginn durchzogen von der Kontroverse um Sinn und Perspektiven der monetären Bewertung. Immerhin ist das Geld nicht nur Kern wirtschaftlichen Strebens, sondern auch ein eminent praktischer, weil gleich machender Bewertungsmaßstab. Tatsächlich findet die monetäre Bewertung vielfältigster Sachen, Leistungen und Vorgänge auf den Märkten ständig statt: Preise, die in Transaktionsaktivitäten der verschiedensten Art täglich millionenfach gefordert und gezahlt werden, sind Ausdruck derartiger Bewertung durch mindestens zwei daran beteiligte Akteure.

Nur ist das noch kein Beleg für die Objektivierbarkeit der monetären Bewertung. Im Gegenteil: Die betriebswirtschaftliche Forschung hat vielfach den Beweis dafür erbracht, dass die monetäre Bewertung von Sachen, die nicht selbst Geld sind, nicht willkürfrei möglich ist, weil diesem Vorgang unaufhebbare zeitliche und sachliche Zurechnungsprobleme im Weg stehen. Unternehmensbilanzen weisen den Jahresgewinn bis auf die Stellen hinter dem Komma genau in einem Geldbetrag aus und basieren doch auf einer Vielzahl willkürlicher Bewertungsakte von Vermögens- und Kapitalposten der Bilanz. Verschärft gilt diese Problematik für außermarktliche Phänomene, und die sind im sozial-ökologischen Bereich die Regel. 
Andererseits führt der Verzicht auf eine aggregierende Bewertung ursprünglich unterschiedlich dimensionierter Detailgrößen zu praktisch unbrauchbaren Informationsfriedhöfen, wenn das zu bewertende Phänomen nur etwas komplexer ist. Daher ist gerade in der sozial-ökologischen Bewertung immer wieder nach Geldsubstituten wie Ökopunkten und dergleichen gesucht worden: Ruedi Müller-Wenks Ökologische Buchhaltung von 1978 war nur der erste Versuch auf diesem Felde.

Die Bemühungen haben ihren Reiz, aber auch ihre Probleme: Sie bieten eine handhabbare Entscheidungsgrundlage, aber sie vernichten Information, wie die ökonomische Modelltheorie schon wesentlich länger weiß: „Bei zu geringer Aggregation bleiben mehr Details bestehen, als wir bearbeiten können. ... Bei zu weitgehender Aggregation werden Tatbestände zusammengefasst, die nichts miteinander $\mathrm{zu}$ tun haben." (1)

\section{- Perspektiven}

Fasst man die dargestellten Probleme zusammen, dann scheint die Schlussfolgerung unabweisbar, dass die valide sozial-ökologische Unternehmensbewertung eine Fiktion darstellt, der die Realität niemals gerecht werden kann und wird. Unternehmensinformation über wirtschaftliche Angelegenheiten, erst recht aber über die sozialen und ökologischen Folgen der Unternehmenstätigkeit wird immer mehr $\mathrm{zu}$ einem Imageeintopf, in dem viele Köche herumrühren. Dabei lassen sich seine Zutaten immer weniger herausschmecken.

Dennoch kann es keine Alternative dazu geben, sich in möglichst differenzierter Weise um Problemlösungen zu bemühen. Kristallkugel und Kaffeesatz sind allemal weniger verlässliche Entscheidungs- und Handlungsgrundlagen.

Erste und wichtigste Voraussetzung dafür sind valide und transparente Informationsgrundlagen des Unternehmens selbst. Hierzu ist eine standardisierte und verpflichtende regelmäßige Nachbaltigkeitsberichterstattung wesentliche Grundlage. Im ersten Entwurf zum Umweltgesetzbuch war eine solche Regelung - dort allerdings allein auf Umweltschutzbelange bezogen - bereits einmal nahe an der Verwirklichung. Bisher dagegen vorgebrachte Argumente, dazu fehlten die wissenschaftlichen Grundlagen und Erfahrungen, treffen heute nicht mehr zu.

Was allerdings zutrifft - heute mehr denn je ist, dass zumindest der Umweltberichterstattung in Form von Umwelterklärungen nach dem EGÖko-Audit-System von Seiten der Adressaten immer geringeres öffentliches Interesse entgegengebracht wird. Hier kommen die offensichtlich notwendigen Mittler ins Spiel: Informations- und Bewertungsagenten müssen die Unternehmensinformationen aufbereiten und einer aggregierenden Bewertung zugänglich machen. Dazu müssen sie ihrerseits über Charakteristika verfügen, die das unvermeidliche Ausmaß der Informationsfärbung und -verzerrung begrenzen: Neutralität und wirtschaftliche Unabhängigkeit vom Informanten sind die wichtigsten.

Nicht zuletzt gibt es auch Anforderungen an die Stakeholder als Informationsempfänger. Diese betreffen vor allem deren Informationsinteresse und -engagement. Sowohl die Bemühungen der Unternehmen als Informanten als auch der Intermediäre als Informationsbearbeiter und -mittler laufen leer, wenn ihre Informationsleistungen nicht oder nur in geringem Umfang nachgefragt werden.

Hier nun schließt sich der Kreis: Stakeholder werden nur dann an sozial-ökologischer Unternehmensinformation interessiert sein, wenn sie tatsächlich substanzielle Möglichkeiten zur Interessenartikulation und -durchsetzung finden. Die reale Entwicklung der corporate governance im Spannungsfeld von Globalisierung und sozialer Verankerung ist entscheidend auch für die Entwicklung und Bereitstellung der notwendigen Informationsgrundlagen. Vielleicht wirkt dabei sogar das Informationsinteresse der internationalen Kapitalmarktagenten als Katalysator eines Prozesses, von dem auch die Öffentlichkeit, die Kunden und andere externe Stakeholder profitieren können.

\section{Anmerkung}

(1) Morgenstern, Oskar: Über die Genauigkeit wirtschaftlicher Beobachtungen, Wien 1965, S. 102.

\section{Der Autor}

Dr. Jürgen Freimann ist Professor für betriebliches Umweltmanagement an der Universität Gesamthochschule Kassel.

Kontakt: Nora-Platiel-Str. 5, 34127 Kassel.

Tel. 0561/ 804-3082, Fax -7739,

E-mail: freimann@wirtschaft.uni-kassel.de

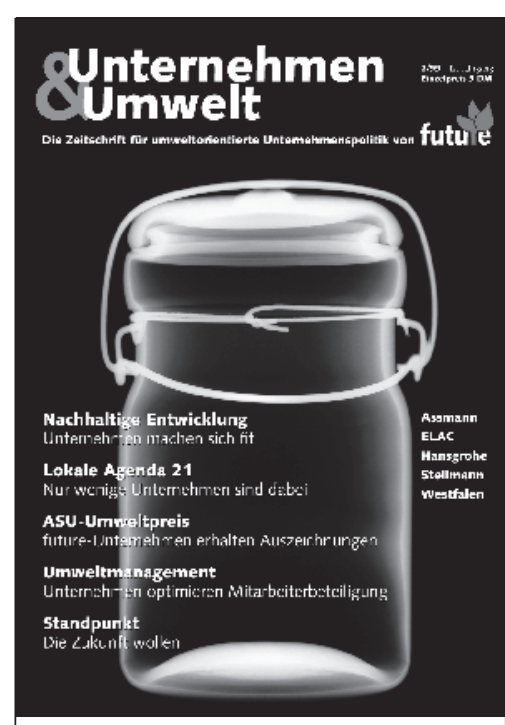

Unternehmen \& Umwelt

- ist die Zeitschrift für umweltorientierte Unternehmenspolitik von future e.V.,

- berichtet über den neuesten Stand zum betrieblichen Umweltmanagement in Theorie und Praxis,

- bietet vier Mal im Jahr ein Schwerpunktthema, Unternehmensbeispiele, Literaturund Veranstaltungshinweise und aktuelle future-Nachrichten.

Zum Beispiel Heft 2/99:

\section{Nachhaltigkeit}

- Nachhaltige Entwicklung: Unternehmen machen sich fit.

- Lokale Agenda 21: Nur wenige Unternehmen sind dabei.

- Betrieblicher Umweltschutz: Assman, ELAC, Hansgrohe, Steilmann.

- Umweltmanagement: Unternehmen optimieren die Mitarbeiterbeteiligung.

Fordern Sie Ihr kostenloses

Probeexemplar an:

Fon: 0234 - 97995 - 13

Fax: 0234 - 97995 - 14

future e.V. Büro Bochum

Am Varenholt 123

44797 Bochum

email: future@umis.de 
(c) 20I0 Authors; licensee IÖW and oekom verlag. This is an article distributed under the terms of the Creative Commons Attribution Non-Commercial No Derivates License (http://creativecommons.org/licenses/by-nc-nd/3.o/), which permits unrestricted use, distribution, and reproduction in any medium, provided the original work is properly cited. 